\title{
Solvent-Free Michael Addition Between EMME and Secondary Amine under Focused Microwave Irradiation
}

\author{
Ki Won Kim, ${ }^{\dagger}$ Hee Jung Lee, ${ }^{\dagger}$ Jeong Im Jo, ${ }^{\dagger}$ and Tae Woo Kwon ${ }^{\dagger, * *}$ \\ ${ }^{\dagger}$ Department of Chemistry and ${ }^{\ddagger}$ Department of Chemical Education, Kyungsung University, Busan 608-736, Korea \\ *E-mail: twkwon@ks.ac.kr \\ Received November 6, 2009, Accepted February 24, 2010
}

\begin{abstract}
Microwave-assisted Michael reaction between EMME and various amines such as diphenylamine, 4-methyl- $N$-phenylbenzenamine, $N$-phenylnaphthalen-1-amine, dihexylamine, diisopropylamine, and 4-nitrobenzenamine were described. Solvent-free conditions on alumina as solid support in the presence of $\mathrm{K}_{2} \mathrm{CO}_{3}$ catalysts gave moderate to good yields (55-93\%) of diethylmalonate analogues having enamine moieties under focused microwave irradiation.
\end{abstract}

Key Words: Focused microwave-assisted irradiation, Solvent-free Michael addition, Diethyl ethoxymethylenemalonate, Secondary amines, Diethyl 2-((diaryl or dialkyl lamino) methylene) malonate

\section{Introduction}

Since the first antimicrobial quinolone was discovered about several decades ago, variety of quinolone analogues $\mathbf{1}$ have been used for the treatment of various infectious diseases. ${ }^{1}$ Some of the enamine type compounds showed interesting central nervous system activity and muscle relaxing properties. For example, $N$-diphenyl compounds, $2\left(\mathrm{R}_{1}=\right.$ phenyl $)$ have shown to be potentially useful intermediates for prevention and treatment of hyperlipemia and atherosclerosis. ${ }^{2}$ Cyclization of various enamines $\mathbf{2}$ directly led to the important building block of quinolones, 1 which shows anti gramnegative antibacterial activity. ${ }^{3}$<smiles>[R]c1cc2c(=O)c(C(=O)O)cn([R1])c2cc1[R]</smiles>

1

$\mathrm{R}_{1}=$ alkyl
$R_{2}$ and $R_{3}=F, H$<smiles>[X]C([X])=CN([R7])c1ccccc1</smiles>

2

$\mathrm{X}, \mathrm{Y}=\mathrm{CO}_{2} \mathrm{Et}, \mathrm{CN}, \mathrm{COCH}_{3}$ $\mathrm{R}_{1}=\mathrm{H}$ or aryl
The Michael addition reaction provides a convenient method for the preparation of a number of useful synthetic intermediates. Current growing research interest is the use of attractive acceptors for a variety of Michael addition donors and a number of quinolone syntheses have been reported. ${ }^{4}$ Since diethyl ethoxymethylenemalonate (EMME) contains an electron-deficient alkene, it can be used as a very attractive acceptor for a variety of Michael addition donors. ${ }^{5}$ In general, these reactions were carried out according to a procedure in which the reaction of amine $N$-nucleophiles with electron-deficient EMME compounds resulted in the displacement of the ethoxy group by the amino function to give important nitrogen-containing precursors in the field of medicinal chemistry. ${ }^{6}$ In the case of the Michael addition reactions between aminoalditol and EMME, the reaction times usually take more than $24 \mathrm{hr}$ under basic conditions in methanol solvent during the synthesis of carbohydrate-based molecules. ${ }^{7}$ As well as using toxic solvents, in some cases, 54 hour of reaction time is necessary for the Michael addition between primary amine such as tryptamine and EMME for the synthesis of 4-quinolone derivatives which are closely related to brain $\mathrm{GABA}_{\mathrm{A}}$ receptors. ${ }^{8}$

In recent years a tremendous number of scientifically focused microwave enhanced organic reactions have attracted a considerable growing interest. The associated literature has been greatly extended due to facile control of the reaction temperature, pressure, power and reaction times with a high degree of reproducibility. ${ }^{9}$ Solid supports such as aluminas, silica gels and other inorganic materials appeared to be valuable to enhance the reaction rates and to carry facile workup procedure and purification. We have studied previously that a variety of reactions such as Knoevenage ${ }^{10}$ condensation and Friedlander type $^{11}$ quinoline synthesis can be performed by solvent-free microwave irradiation technique. More recently, we have been able to demonstrate the microwave enhanced Michael addition between various nucleophiles $\left(-\mathrm{OH},-\mathrm{SH}\right.$ and $\left.-\mathrm{NH}_{2}\right)$ and EMME. $^{12}$ A significant microwave effect and excellent yields were observed in the case of primary amines or $\mathrm{PhSH}$ under microwave irradiation. However, no general Michael addition between secondary amine and electron deficient EMME has been studied under microwave irradiation. Secondary amine associated diethyl methylenemalonate adducts 3-7 clearly play an important role for the synthesis of new biologically important $\mathrm{N}, \mathrm{N}$-disubstituted quinolone, $\mathbf{1}$.

Our aim is to develop fast and efficient synthetic routes to achieve $N, N$-disubstituted enamine analogues using solvent free microwave irradiation technique. Herein, we focused on the microwave assisted Michael addition reaction between EMME and various secondary amines in the presence of $\mathrm{K}_{2} \mathrm{CO}_{3}$ catalysts on alumina as solid support.

\section{Results and Discussion}

First attempts were carried out to find the optimized reaction condition using EMME and diphenyl amine under monomode 
Table 1. Optimization of the reaction ${ }^{a}$ time at $100{ }^{\circ} \mathrm{C}$ in the solvent-free Michael Addition between EMME and diphenylamine ${ }^{b}$ under MWI

\begin{tabular}{|c|c|c|c|c|}
\hline Entry & $\begin{array}{l}\text { support } \\
\left(\mathrm{Al}_{2} \mathrm{O}_{3}\right)\end{array}$ & $\begin{array}{l}\text { catalyst } \\
\left(\mathrm{K}_{2} \mathrm{CO}_{3}\right)\end{array}$ & $\begin{array}{l}\text { irradiation time } \\
\qquad(\mathrm{min})\end{array}$ & yield $(\%)^{d}$ \\
\hline 1 & none & none & 2 & trace \\
\hline 2 & $0.12 \mathrm{~g}$ & none & 2 & 17 \\
\hline 3 & $0.12 \mathrm{~g}$ & $0.19 \mathrm{~g}$ & 2 & 22 \\
\hline 4 & $0.12 \mathrm{~g}$ & $0.19 \mathrm{~g}$ & 4 & 19 \\
\hline 5 & $0.12 \mathrm{~g}$ & $0.19 \mathrm{~g}$ & 10 & 25 \\
\hline 6 & $0.12 \mathrm{~g}$ & $0.19 \mathrm{~g}$ & 20 & 62 \\
\hline 7 & $0.12 \mathrm{~g}$ & $0.19 \mathrm{~g}$ & 40 & 70 \\
\hline
\end{tabular}

${ }^{a}$ Reaction of $5.55 \mathrm{mmol}$ scale, ${ }^{b}$ Mole ratio of EMME: diphenyl amine $=$ 1:1.3 equiv. ${ }^{c} \mathrm{CEM}$ Focused Microwave ${ }^{\mathrm{TM}}$ Synthesis System equipped with a reflux condenser. ${ }^{d}$ Yields were not optimized and based on starting EMME.

Table 2. Optimization of the reaction temperature for the synthesis ${ }^{a}$ of diethyl 2-((diphenylamino)methylene)malonate in the presence of $\mathrm{Al}_{2} \mathrm{O}_{3}$ and $\mathrm{K}_{2} \mathrm{CO}_{3}$ catalyst ${ }^{b}$

\begin{tabular}{cccc}
\hline Entry & $\begin{array}{c}\text { irradiation time } \\
(\mathrm{min})\end{array}$ & $\begin{array}{c}\text { temperature } \\
\left({ }^{\circ} \mathrm{C}\right)\end{array}$ & yield(\%) ${ }^{d}$ \\
\hline 1 & 4 & 100 & 19 \\
2 & 5 & 150 & 20 \\
3 & 5 & 175 & 68 \\
4 & 5 & 200 & 93 \\
\hline
\end{tabular}

${ }^{a}$ Reaction of $5.55 \mathrm{mmol}$ scale, ${ }^{b}$ Equivalent ratio of support $\mathrm{Al}_{2} \mathrm{O}_{3}$ : catalyst $\mathrm{K}_{2} \mathrm{CO}_{3}=1.3: 1.5$, mole ratio of EMME: diphenyl amine $=1: 1.3$ equiv. ${ }^{c}$ CEM Focused Microwave ${ }^{\mathrm{TM}}$ Synthesis System equipped with a reflux condenser. ${ }^{d}$ Isolated yields after purification.

microwave irradiation in an solvent-free condition. Several efforts have been conducted using various reaction conditions at $100^{\circ} \mathrm{C}$. In the absence of support and catalyst, Michael addition did not undergo (Table 1, entry 1). When basic catalyst $\mathrm{K}_{2} \mathrm{CO}_{3}$ was used in the presence of solid support, the reaction rates were slightly increased but generally very slow within short period of 2 - 10 mins (Table 1, entries 2-5).

Clearly, longer irradiation time gave better yields. When the reaction mixtures were irradiated for 10,20 and 40 min respectively, the yields were increased upto $70 \%$ at $100{ }^{\circ} \mathrm{C}$ (Table 2, entries 2, 3 and 4). More interestingly, noticeable enhancements in both reaction rate and yields were observed when the temperature was increased up to $200{ }^{\circ} \mathrm{C}$ as shown in Table 2. The maximum yield of $93 \%$ was obtained within 5 min (Table 2, entry 4).

Our optimized reaction conditions were extended to synthesize enamine esters using EMME and variety of amines such as diphenylamine, 4-methyl- $N$-phenylbenzenamine, $N$-phenylnaphthalen-1-amine, dihexylamine and diisopropylamine (Table 3 , entries 1-5). In the case of aniline having electron-withdraw-
Table 3. Michael addition reaction between EMME and various amine under microwave irradiation (MWI) and conventional heating in the presence of $\mathrm{Al}_{2} \mathrm{O}_{3} / \mathrm{K}_{2} \mathrm{CO}_{3}$

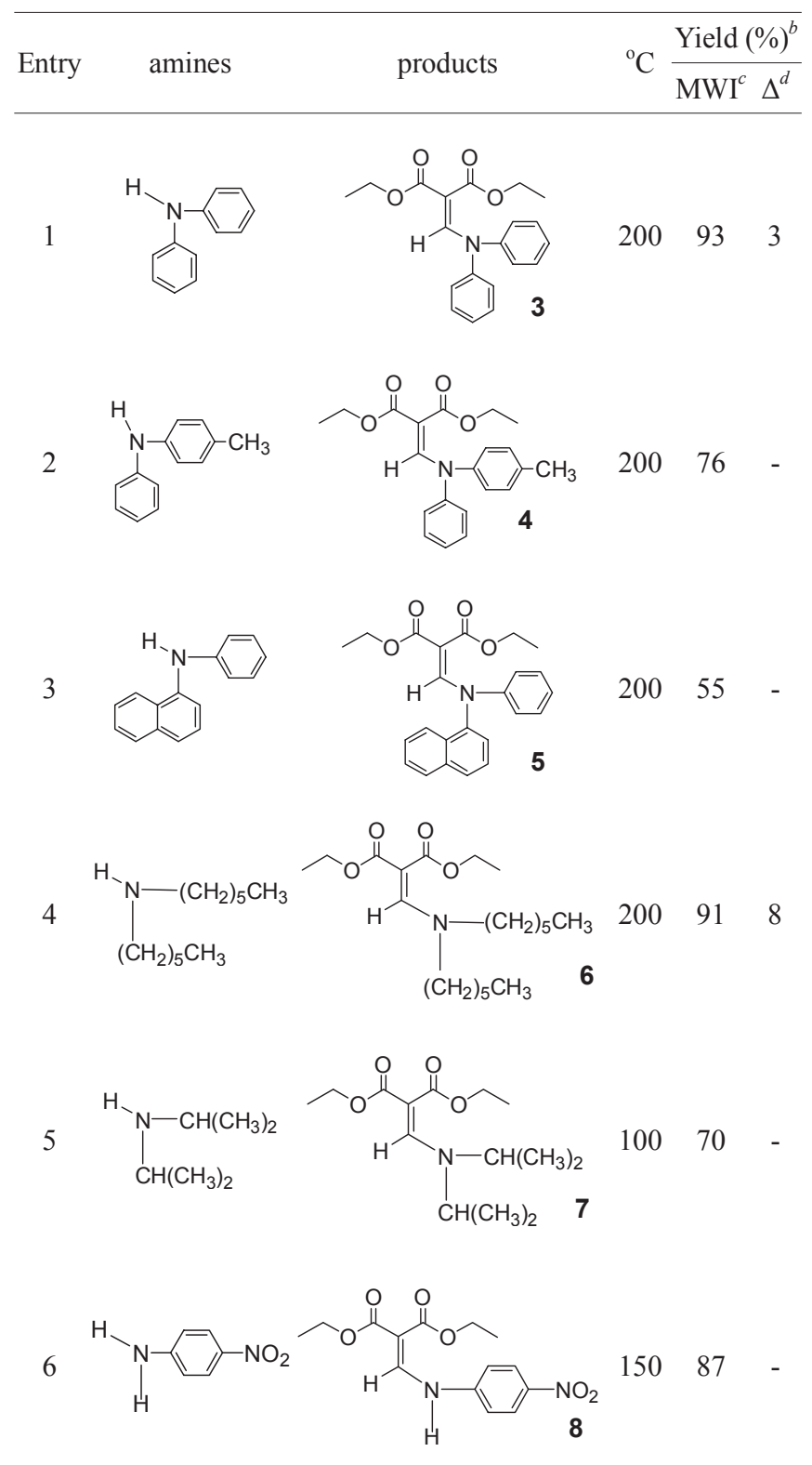

${ }^{a}$ Mole ratio of EMME : amine $=(0.2 \mathrm{~g}, 0.925 \mathrm{mmol}, 1$ eq. $):(1.20 \mathrm{mmol}, 1.3$ eq.). ${ }^{b}$ Isolated yields after purification. All products were characterized by ${ }^{\mathrm{H}}$ and ${ }^{13} \mathrm{C}$ NMR, IR, MS. ${ }^{c} \mathrm{CEM}$ Focused Microwave ${ }^{\mathrm{TM}}$ Discover equipped with a reflux condenser. ${ }^{d} \mathrm{~A}$ thermostated oil bath heating at the same temperature of MWI.

ing nitro group at the para-position, the reaction also proceeded smoothly even at $150^{\circ} \mathrm{C}$ in good yield and no side product was detected by TLC (Table 3, entry 6). In all cases, moderate to excellent yields were obtained in the absence of solvent.

After the successful performance of Michael additions were established under MW irradiation, the microwave heating $v s$ conventional heating, in which pre-heated oil-bath kept at 200 ${ }^{\circ} \mathrm{C}$ were compared in order to see the importance of microwave effect between EMME and various secondary nucleophilic functionality such as diphenyl amine and dihexyl amine. In both 
cases, yields obtained with MW irradiation were dramatically improved (93 vs 3, 91 vs 8\%) when compared to oil-bath experiments conducted at the same temperature (Table 3, entries 1 and 4). This observation demonstrates that the effect of MW irradiation is not purely thermal.

All final compounds were characterized by ${ }^{1} \mathrm{H},{ }^{13} \mathrm{C}$ NMR, IR and mass spectral data which were fully in accord with expected structures. It has been emphasized that microwave has a tendency to stabilize the more polar transition states (TS) to a greater extent than the less polar ground state (GS). ${ }^{13}$ As proposed in the mechanism, the transition state between EMME and amines by dipole-dipole electrostatic interactions is involved in the reaction mechanism. The enhanced reaction progress under microwave condition can be explained to the fact that the reaction intermediates (TS), $\mathbf{1 0}$ is more polar than the starting materials in the ground state (GS), 9.

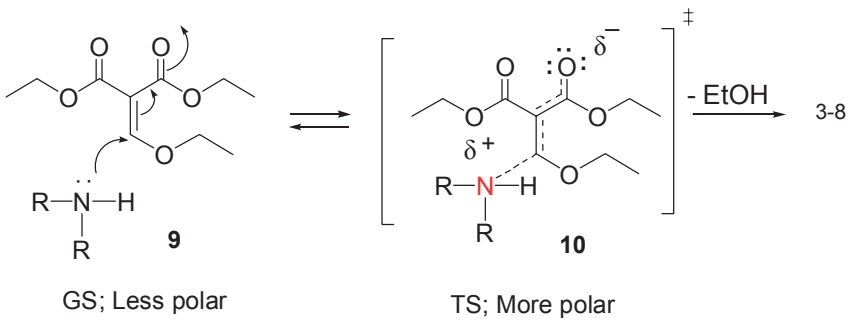

Summary

We have described the environmentally benign Michael addition of various secondary amine nucleophiles leading to 3-7 having enamine bond formation under MWI. Deactivated nitroaniline also gave high yield of $\mathbf{8}$ within 5 minutes of short irradiation time. A rapid and economical method using inexpensive and non-toxic $\mathrm{Al}_{2} \mathrm{O}_{3}$ in the absence of solvent has been demonstrated under MW irradiation. Further investigations regarding the Microwave enhanced synthesis of variety of quinolones are currently under progress.

\section{Experimental Section}

All chemicals were obtained from Sigma-Aldrich (Korea) and used as received. TLC was performed on pre-coated glass plate-silica gel 250-m (Baker Si 250F) with detection by UV light. Flash column chromatography was performed on silica gel (230 - 400 mesh). The ${ }^{1} \mathrm{H}$ and ${ }^{13} \mathrm{C}$ NMR spectra were recorded at $300 \mathrm{MHz}$ and $75 \mathrm{MHz}$ on a FT-NMR Bruker 300. Chemical shifts are given in ppm and referenced to internal tetramethylsilane (TMS, $\delta=0 \mathrm{ppm}$ ) standard. GC/MS spectra were measured on a Shimazu QP 5000 spectrometer. Microwave irradiation was carried out by a CEM Discover focused microwave $(2450 \mathrm{MHz}, 300 \mathrm{~W})$ under atmospheric pressure.

General microwave procedure for microwave enhanced Michael addition between EMME and amines; Synthesis of diethyl 2-((diphenylamino)methylene)malonate (Table 3, entry 1). EMME (0.20 g, 0.93 mmol, 1.0 equiv.), diphenylamine ( $0.20 \mathrm{~g}$, 1.21 mmol, 1.3 equiv. $), \mathrm{K}_{2} \mathrm{CO}_{3}(0.19 \mathrm{~g}, 1.40 \mathrm{mmol}, 1.5$ equiv. $)$ and $\mathrm{Al}_{2} \mathrm{O}_{3}(0.12 \mathrm{~g}, 0.93 \mathrm{mmol}, 1.0$ equiv.) were mixed together in a microwave tube with a magnetic stirrer bar in the absence of any organic solvent. The mixture was then submitted for 5 min to microwave irradiation inside a CEM microwave oven (temperature : $200{ }^{\circ} \mathrm{C}$, pressure : 80PSI, hold time : 3 min, Run time : $5 \mathrm{~min}$, Power : $150 \mathrm{w}$ ) and monitored by thin-layer chromatography. The reaction mixture was dissolved in ethyl acetate and insoluble solids were filtered off. The filtrate was concentrated by rotary vaccum evaporator. The crude product was purified by column chromatography using silica gel (10\% ethylacetate $/ 90 \%$ hexane: $\mathrm{v} / \mathrm{v}, R_{f}=0.6$ ) to give $\mathbf{3}$ as a colorless oil (0.29 g, $0.85 \mathrm{mmol}, 92 \%$ ). ${ }^{\mathrm{H}} \mathrm{NMR}\left(300 \mathrm{MHz}, \mathrm{CDCl}_{3}\right) \delta 7.95$ (1H, s, vinyl H), 7.33 (4H, m, ortho-Hs), 7.21 (2H, m, para-Hs), 7.10 (4H, m, meta-Hs), $4.20\left(2 \mathrm{H}, \mathrm{q}, \mathrm{OCH}_{2}\right), 3.44\left(2 \mathrm{H}, \mathrm{q}, \mathrm{OCH}_{2}\right)$, $1.27\left(3 \mathrm{H}, \mathrm{t}, \mathrm{CH}_{3}\right) .1 .04\left(3 \mathrm{H}, \mathrm{t}, \mathrm{CH}_{3}\right) .{ }^{13} \mathrm{CNMR}\left(75 \mathrm{MHz}, \mathrm{CDCl}_{3}\right)$ $166.65(\mathrm{C}=\mathrm{O}), 165.28(\mathrm{C}=\mathrm{O}), 146.39,129.31,126,34,124.51$, $102.88,60.47\left(\mathrm{OCH}_{2}\right), 60.38\left(\mathrm{OCH}_{2}\right), 14.31\left(\mathrm{CH}_{3}\right)$ and 13.77 $\left(\mathrm{CH}_{3}\right) \mathrm{ppm}$. IR (neat) 3010, 2919, 1720, 1695, 1575, 1490, 1361, $1189,1066,753,695 \mathrm{~cm}^{-1}$. GC/MS $339\left(\mathrm{M}^{+}\right)$.

Diethyl 2-((phenyl(p-tolyl)amino)methylene)malonate (Table 3, entry 2). Prepared by the general procedure using EMME ( $0.20 \mathrm{~g}, 0.93 \mathrm{mmol}, 1.0$ equiv. $), 3$-methyldiphenyl-amine (0.22 g, 1.21 mmol, 1.3 equiv.), $\mathrm{Al}_{2} \mathrm{O}_{3}(0.12 \mathrm{~g}, 0.93 \mathrm{mmol}$, 1.0 equiv.) and $\mathrm{K}_{2} \mathrm{CO}_{3}(0.19 \mathrm{~g}, 1.40 \mathrm{mmol}, 1.5$ equiv.) gives 4 as a colorless oil ( $76 \%$ yield, $0.25 \mathrm{~g} 0.71 \mathrm{mmol})$ after purification by column chromatography using silica gel (10\% ethylacetate/ $90 \%$ hexane $\left.: \mathrm{v} / \mathrm{v}, R_{f}=0.6\right) .{ }^{1} \mathrm{H}$ NMR $\left(300 \mathrm{MHz}, \mathrm{CDCl}_{3}\right) \delta 7.95$ (1H, s, vinyl H), $7.32(2 \mathrm{H}, \mathrm{m}), 7.22(2 \mathrm{H}, \mathrm{m}), 7.19(2 \mathrm{H}, \mathrm{m}), 7.11$ $(1 \mathrm{H}, \mathrm{m}), 7.08(1 \mathrm{H}, \mathrm{m}), 6.93(1 \mathrm{H}, \mathrm{m}), 4.22(2 \mathrm{H}, \mathrm{q}), 3.46(2 \mathrm{H}, \mathrm{q})$, $2.32(3 \mathrm{H}, \mathrm{s}), 1.27\left(3 \mathrm{H}, \mathrm{t}, \mathrm{CH}_{3}\right)$, and $1.05\left(3 \mathrm{H}, \mathrm{t}, \mathrm{CH}_{3}\right) .{ }^{13} \mathrm{C} \mathrm{NMR}$ $\left(75 \mathrm{MHz}, \mathrm{CDCl}_{3}\right) 166.56(\mathrm{C}=\mathrm{O}), 165.16(\mathrm{C}=\mathrm{O}), 146.32,144.38$, 139.23, 129.14., 128.99, 126.94, 126.04, 124.99, 124.33, $121.15,102.49,60.29,60.22,21.13,14.20$ and 13.65 ppm. IR (neat) 3015, 2975, 2832, 1722, 1694, 1574, 1490, 1381, 1177, $1071 \mathrm{~cm}^{-1}$. GC/MS $353\left(\mathrm{M}^{+}\right)$.

Diethyl 2-((naphthalen-1-yl(phenyl)amino)methylene)malonate (Table 3, entry 3). Prepared by the general procedure from EMME (0.20 g, 0.93 mmol, 1.0 equiv. $), n$-phenyl-1-naphtylamine ( $0.26 \mathrm{~g}, 1.21 \mathrm{mmol}, 1.3$ equiv. $), \mathrm{Al}_{2} \mathrm{O}_{3}(0.12 \mathrm{~g}, 0.93 \mathrm{mmol}$, 1.0 equiv.) and $\mathrm{K}_{2} \mathrm{CO}_{3}(0.19 \mathrm{~g}, 1.40 \mathrm{mmol}, 1.5$ equiv.) gives 5 as a white solid. $\left(0.198 \mathrm{~g}, 0.51 \mathrm{mmol}, 55 \%, \mathrm{mp}=129 \sim 131{ }^{\circ} \mathrm{C}\right)$ after purification by column chromatography using silica gel (10\% ethylacetate/90\% hexane). ${ }^{1} \mathrm{H} \mathrm{NMR}\left(300 \mathrm{MHz}, \mathrm{CDCl}_{3}\right)$, $\delta 8.25(1 \mathrm{H}, \mathrm{s}$, vinyl $\mathrm{H}), 7.89(2 \mathrm{H}, \mathrm{m}), 7.75(1 \mathrm{H}, \mathrm{m}), 7.51-7.47$ $(4 \mathrm{H}, \mathrm{m}), 7.26(2 \mathrm{H}, \mathrm{m}), 7.12(1 \mathrm{H}, \mathrm{m}), 7.01(2 \mathrm{H}, \mathrm{m}), 4.20(2 \mathrm{H}, \mathrm{q})$, $3.29(1 \mathrm{H}$, broad q), $2.65(1 \mathrm{H}$, broad q) $1.23(3 \mathrm{H}, \mathrm{t}), 0.62(3 \mathrm{H}$, t). ${ }^{13} \mathrm{C} \mathrm{NMR}\left(75 \mathrm{MHz}, \mathrm{CDCl}_{3}\right) 166.46(\mathrm{C}=\mathrm{O}), 165.14(\mathrm{C}=\mathrm{O})$, $146.56,146.41,134.55,129.35,129.84,128.30,127.15,126.52$, $125.50,124.82,123.46,120.41,102.90,68.44,68.14,14.25$ and $13.09\left(\mathrm{CH}_{3}\right) \mathrm{ppm}$. IR (neat) 3059, 2976-2869, 1717, 1686, $1566,1486,1381,1295,1169,1071,766$ and $695 \mathrm{~cm}^{-1}$. GC/MS $389\left(\mathrm{M}^{+}\right)$.

Diethyl 2-((dihexylamino)methylene)malonate (Table 3, entry 4). Prepared by the general procedure from $\operatorname{EMME~}(0.20 \mathrm{~g}$, $0.93 \mathrm{mmol}, 1.0$ equiv.), dihexylamine ( $0.22 \mathrm{~g}, 1.21 \mathrm{mmol}, 1.3$ equiv.), $\mathrm{Al}_{2} \mathrm{O}_{3}$ (0.12 g, $0.93 \mathrm{mmol}, 1.0$ equiv.) and $\mathrm{K}_{2} \mathrm{CO}_{3}$ $(0.19 \mathrm{~g}, 1.40 \mathrm{mmol}, 1.5$ equiv. ) gives 6 as a colorless oil $(0.3 \mathrm{~g}$, $0.84 \mathrm{mmol}, 91 \%$ ) after purification by column chromatography using silica gel (10\% ethylacetate/90\% hexane). ${ }^{1}$ H NMR (300 
$\left.\mathrm{MHz}, \mathrm{CDCl}_{3}\right) \delta 7.27(1 \mathrm{H}, \mathrm{s}), 4.03(4 \mathrm{H}$, broad q), $3.05(4 \mathrm{H}$, broad t), $1.39(4 \mathrm{H}, \mathrm{m}), 1.12(16 \mathrm{H}, \mathrm{m})$ and $0.72(6 \mathrm{H}$, two t $) .{ }^{13} \mathrm{C} \mathrm{NMR}$ $\left(75 \mathrm{MHz}, \mathrm{CDCl}_{3}\right) 167.51(\mathrm{C}=\mathrm{O}), 167.38(\mathrm{C}=\mathrm{O}), 150.27,92.31$, 60.22, 59.34, 58.27, 49.13, 31.10, 28.94, 26.76, 25.81, 22.13, 13.82 and $13.55 \mathrm{ppm}$. IR (neat) 2957-2852, 1681, 1594, 1462, $1382,1253,1212,1180,1125,1101,1066,1034,857$ and 756 $\mathrm{cm}^{-1}$. GC/MS $383\left(\mathrm{M}^{+}\right)$.

Diethyl 2-((diisopropylamino)methylene)malonate (Table 3, entry 5). Prepared by the general procedure using EMME (0.20 g, $0.93 \mathrm{mmol}, 1.0$ equiv.), diisopropylamine ( $0.12 \mathrm{~g}, 1.21 \mathrm{mmol}$, 1.3 equiv.), $\mathrm{Al}_{2} \mathrm{O}_{3}$ ( $0.12 \mathrm{~g}, 0.93$ mmol, 1.0 equiv.) and $\mathrm{K}_{2} \mathrm{CO}_{3}$ $(0.19 \mathrm{~g}, 1.40 \mathrm{mmol}, 1.5$ equiv. $)$ gives 7 as a colorless oil $(0.18 \mathrm{~g}$, $0.65 \mathrm{mmol}, 70 \%$ ) after purification by column chromatography using silica gel (20\% ethylacetate/80\% hexane). ${ }^{1} \mathrm{H}$ NMR (300 $\left.\mathrm{MHz} \mathrm{CDCl}_{3}\right) \delta 7.36(1 \mathrm{H}, \mathrm{s}), 4.11(4 \mathrm{H}, \mathrm{dd}), 2.95(4 \mathrm{H}, \mathrm{dd}), 1.78$ $(2 \mathrm{H}$, two septet $), 1.21(3 \mathrm{H}, \mathrm{t}), 1.14(3 \mathrm{H}, \mathrm{t})$ and $0.77-0.76(12 \mathrm{H}$, dd). ${ }^{13} \mathrm{C} \mathrm{NMR}\left(75 \mathrm{MHz}, \mathrm{CDCl}_{3}\right) 167.61(\mathrm{C}=\mathrm{O}), 167.20(\mathrm{C}=\mathrm{O})$, $92.73,60.27,59.57,50.70,28.16,25.16,19.43,14.15$ and 14.03 ppm. IR (neat) 2955-2869, 1678, 1590, 1463, 1360, 1242, 1183, $1127,1063,960,859$ and $759 \mathrm{~cm}^{-1}$. GC/MS $271\left(\mathrm{M}^{+}\right)$.

Diethyl 2-((4-nitrophenylamino)methylene)malonate (Table 3, entry 6). Prepared by the general procedure from EMME ( $0.20 \mathrm{~g}, 0.93 \mathrm{mmol}, 1.0$ equiv.), 4-nitroaniline $(0.17 \mathrm{~g}, 1.21$ mmol, 1.3 equiv.), $\mathrm{Al}_{2} \mathrm{O}_{3}$ ( $0.12 \mathrm{~g}, 0.93 \mathrm{mmol}, 1.0$ equiv.) and $\mathrm{K}_{2} \mathrm{CO}_{3}(0.19 \mathrm{~g}, 1.40 \mathrm{mmol}, 1.5$ equiv. $)$ gives 8 as a yellow solid. $\left(0.25 \mathrm{~g}, 0.81 \mathrm{mmol}, 87 \%, \mathrm{mp}=127 \sim 135^{\circ} \mathrm{C}\right)$ after purification by column chromatography using silica gel ( $20 \%$ ethylacetate/ $80 \%$ hexane). ${ }^{1} \mathrm{H} \mathrm{NMR}\left(300 \mathrm{MHz}, \mathrm{CDCl}_{3}\right) \delta 11.17(1 \mathrm{H}, \mathrm{d}, J=$ $9.75 \mathrm{~Hz}), 8.48(1 \mathrm{H}, J=9.99 \mathrm{~Hz}$, vinyl H), $8.25(2 \mathrm{H}, \mathrm{d}, J=6.7$ $\mathrm{Hz}), 7.20(2 \mathrm{H}, \mathrm{d}, J=6.7 \mathrm{~Hz}), 4.27(4 \mathrm{H}, \mathrm{dd}), 1.35(6 \mathrm{H}, \mathrm{tt}) \mathrm{ppm}$. ${ }^{13} \mathrm{C}$ NMR (75 MHz, $\left.\mathrm{CDCl}_{3}\right) 169.49(\mathrm{C}=\mathrm{O}), 165.03(\mathrm{C}=\mathrm{O})$, 149.64, 144.51, 143.83, 125.95, 116.31, 97.34, 60.91, 60.61, 14.31 and 14.14 ppm. IR (neat) 3070, 2977-2852, 1681, 1574 , $1510,1450,1332,1229,1097,1022,855,802$ and $745 \mathrm{~cm}^{-1}$. GC/MS $308\left(\mathrm{M}^{+}\right)$.

Acknowledgments. This research was supported by the Kyungsung University Research Grants in 2010.

\section{References}

1. (a) Lesher, G. Y.; Froelich, E. J.; Gruett, M. D.; Bailey, J. H.; Brundage, R. P. J. Med. Pharm. Chem. 1962, 91, 1063. (b) Mitscher, Lester A. Chem. Rev. 2005, 105, 559. (c) Koga, H.; Ito, A.; Murayama, S.; Suzue, S.; Irikura, T. J. Med. Chem. 1980, 23(12), 1358. (d) Hayakawa, I.; Hiramitsu, T.; Tanaka, Y. Chem. Pharm. Bull. 1984, 32, 4907.(e) Atarashi, S.; Yokahama, S.; Yamazaki, K.; Sakano, K.; Imamura, M.; Hayakawa, I. Chem. Pharm. Bull. 1987, 35, 1896.
2. (a) Santilli, A. A.; Bruce, W. F.; Osdene, T. S. J. Med. Chem. 1963, 7(1), 68. (b) Steck, E. A. J. Org. Chem. 1962, 27, 306.

3. (a) Banerji, B.; Conejo-Garcia, A.; McNeill, L. A.; McDonough, M. A.; Buck, M. R. G.; Hewitson, K. S.; Oldham, N. J.; Schofield, C. J. Chem. Commun. 2005, 5438. (b) Stern, E.; Muccioli, G. G.; Millet, R.; Goossens, J. F.; Farce, A.; Chavatte, P. Poupaert, J. H.; Lambert, D. M.; Depreux, P.; Hénichart, J-P. J. Med. Chem. 2006, 49,70 .

4. (a) Banerji, B.; Conejo-Garcia, A.; McNeill, L. A.; McDonough, M. A.; Buck, M. R. G.; Hewitson, K. S.; Oldham, N. J.; Schofield, C. J. Chem. Commun. 2005, 5438. (b) Desforges, E.; Grysan, A; Oget, N.; Sindt, M.; Mieloszynski, J. Tetrahedron Lett. 2003, 44, 6273. (c) Yadav, J. S.; Reddy, B. V. S.; Baishya, G.; Narsaiah, A. Chemistry Letters 2005, 34, 102.

5. (a) Chezal, J. M.; Moreau, E.; Delmas, G.; Gueiffier, A.; Blache, Y.; Grassy, G.; Lartigue, C.; Chavignon, O.; Teulade, J. C. Tetrahedron Lett. 2003, 44, 467. (b) Deinnin, F.; Buindeau, D.; Sliwa, H. Tetrahedron Lett. 1989, 30(12), 1529.

6. (a) Kawato, H. C.; Nakayama, K.; Inagaki, H.; and Ohta, T. Org. Lett. 2001, 3(22), 3451. (b) Blanco, M. M.; Avendaño, C.; and Menéndez, J. C. Tetrahedron 1997, 53(33), 11465. (c) Forbes, I. T.; Johnson, C. N.; Jones, G. E.; Loudon, J.; Nicholass, J. M.; Thompson, M.; Upton, N. J. Med. Chem. 1990, 33, 2640. (d) Bare, T. M.; McLaren, C. D.; Campbel1, J. B.; Firor, J. W.; Resch, J. F.; Waiters, C. P.; Salama, A. I.; Meinem, B. A.; Patel, J. B. J. Med. Chem. 1989, 32, 2561.

7. Ávalos, M.; Babiano, R.; Cintas, P.; Hursthouse, M. B.; Jiménez, J. L.; Light, M. E. ; Palacios, J. C.; Pérez, E. M. S. Eur. J. Org. Chem. 2006, 657.

8. Lager, E.; Andersson, P.; Nilsson, J.; Pettersson, I.; Oestergaard Nielsen, E.; Nielsen, M.; Sterner, O.; Liljefors, T. J. Med. Chem. 2006, 49(8), 2526.

9. (a) Kappe, C. O. Angew. Chem., Int. Ed. 2004, 43, 6250 and references therein. (b) Perreux, L.; Loupy, A. Tetrahedron 2001, 57, 9199. (c) Kuhnert, N. Angew. Chem., Int. Ed. 2002, 41, 1863. (d) Strauss, C. R. Angew. Chem., Int. Ed. 2002, 41, 3589. (e) Choi, J. Y.; Kim, J.; Jhung, S. H.; Kim, H. K.; Chang, J. S.; Chae, H. K. Bull. Korean. Chem. Soc. 2006, 27(10), 1523. (f) Park, H. J.; Lee, J. C. Bull. Korean Chem. Soc. 2008, 29(4), 856. (g) Park, J. W.; Lee, K. H. Bull. Korean Chem. Soc. 2009, 30(10), 2475. (h) Kim, K. W.; Lee, H. J.; Kim, C. G.; Park, M. J.; Kwon, T. W. Bull. Korean Chem. Soc. 2008, 29(3), 604. (i) Jo, E. A.; Ahn, J. A.; Jun, C. H. Bull. Korean Chem. Soc. 2007, 28(11), 2020.

10. (a) Kim, J. K.; Kwon, P. S.; Kwon, T. W.; Chung, S. K.; Lee, J. W. Syn. Commun. 1996, 26, 535. (b) Kim, S. Y.; Kwon, P. S.; Kwon, T. W.; Chung, S. K.; Chang, Y. T. Syn. Commun. 1997, 27, 533. (c) Kwon, P. S.; Kim, Y. S.; Kang, C. J.; Kwon, T. W.; Chung, S. K.; Chang, Y. T. Syn. Commun. 1997, 27, 4091. (d) Loupy, A.; Song, S. J.; Sohn, S. M.; Lee, Y. M.; Kwon, T. W. J. Chem. Soc. Perkin Trans. 1 2001, 1220.

11. Song, S. J.; Cho, S. J.; Park, D. K.; Kwon, T. W.; Jenekhe, S. A. Tetrahedron Lett. 2003, 44(2), 255.

12. Loupy, A.; Song, S. J.; Cho, S. J.; Park, D. K.; Kwon, T. W. Synth. Commun. 2005, 35, 79.

13. Loupy, A. Microwaves in Organic Synthesis, 2nd ed.; Wiley-VCH: Weinheim (Germany), 2006. 